\title{
Heat of Reaction, Degree of Cure, and Viscosity of Hercules 3501-6 Resin
}

\author{
Woo Il LeE, Alfred C. LoOS, ANd George S. SPRinger \\ Department of Mechanical Engineering and Applied Mechanics \\ The University of Michigan \\ Ann Arbor, Michigan 48109
}

(Received August 11, 1982)

\begin{abstract}
The heat of reaction, degree of cure, and viscosity of Hercules 3501-6 resin were measured using a differential scanning calorimeter and a disc and plate type viscometer. Expression were developed for correlating the rate of degree of cure and the viscosity with the degree of cure. Viscosities calculated by these expressions were compared with data reported previously, and good agreement was found between the present results and the previous data.
\end{abstract}

\section{INTRODUCTION}

\begin{abstract}
Considerable EFFORTS have been made in recent years to model the curing process of fiber reinforced, organic matrix composites [1-3]. Such models relate the temperature and pressure applied during the cure to the thermal, chemical, and physical processes occurring inside the material. The models require a knowledge of the thermal, chemical, and physical properties of the material which affect the curing process. In this investigation three of the relevant properties, namely the heat of reaction, the degree of cure, and the viscosity of Hercules 3501-6 resin were measured. These three properties were studied because of their important roles in the cure. The 3501-6 resin system was selected because of its wide use in industry.
\end{abstract}

\section{EXPERIMENTAL}

In this section, the experimental apparatus and the procedure are described which were used to measure the heat of reaction, the degree of cure, and the viscosity of the resin. 


\section{Heat of Reaction and Degree of Cure}

The heat of reaction and the degree of cure were measured using a PerkinElmer DSC-2 differential scanning calorimeter. Prior to the tests, the DSC temperature (abscissa) and the DSC energy (ordinate) calibrations were checked following the procedure recommended by the manufacturer [4].

A sample ranging in weight from $3 \mathrm{mg}$ to $8 \mathrm{mg}$ was encapsulated in a standard aluminum sample pan. The encapsulated sample was placed in the DSC sample holder and the rate of heat generation from the sample was measured as a function of time. An empty aluminum sample pan and cover was used as a reference. The weight of the sample was measured before and after the test. The weight losses during the tests were found to be negligible. The rate of heat generation $d Q / d t$ was displayed on a strip chart recorder. The tests were performed in two different ways [5]: a) with the sample kept at constant temperature (isothermal scanning), and b) with the sample heated at a constant scanning rate (dynamic scanning). Isothermal tests were performed at eight different temperatures: $400 \mathrm{~K}, 425 \mathrm{~K}, 430 \mathrm{~K}, 440 \mathrm{~K}, 450 \mathrm{~K}, 465 \mathrm{~K}, 470 \mathrm{~K}$, and $475 \mathrm{~K}$. The total time required for a test ranged from $15 \mathrm{~min}$. (at $400 \mathrm{~K}$ ) to $5 \mathrm{~min}$. (at $475 \mathrm{~K}$ ). The time to reach the steady state was less than 30 seconds. The temperature was raised from room temperature to the test temperature at $320 \mathrm{~K} / \mathrm{min}$.

In the experiments where dynamic scanning was applied, the temperature was raised from $320 \mathrm{~K}$ to $590 \mathrm{~K}$ at the rate of $20 \mathrm{~K} / \mathrm{min}$. The heat of reaction was determined from the experiments using dynamic scanning. The rate of heat generation was plotted versus time and the area under the curve was determined (Figure 1). The heat or reaction was calculated by the expression

$$
H_{R}=\int_{0}^{t_{f}}(d Q / d t) d t
$$

where $t_{f}$ is the time required to complete the reaction.

The amount of heat released up to time $t$ was determined using the rate of heat generations measured during isothermal scanning experiments. The rate of heat generation was plotted versus time and the area under the curve provided the amount of heat released (Figure 1)

$$
H=\int_{0}^{t}(d Q / d t) d t
$$

The degree of cure is defined as

$$
\alpha=H / H_{R}
$$

The rate of degree of cure is calculated by the expression

$$
d \alpha / d t=(d Q / d t) / H_{R}
$$

where the rate of heat generation $d Q / d t$ is provided by the measurements. 

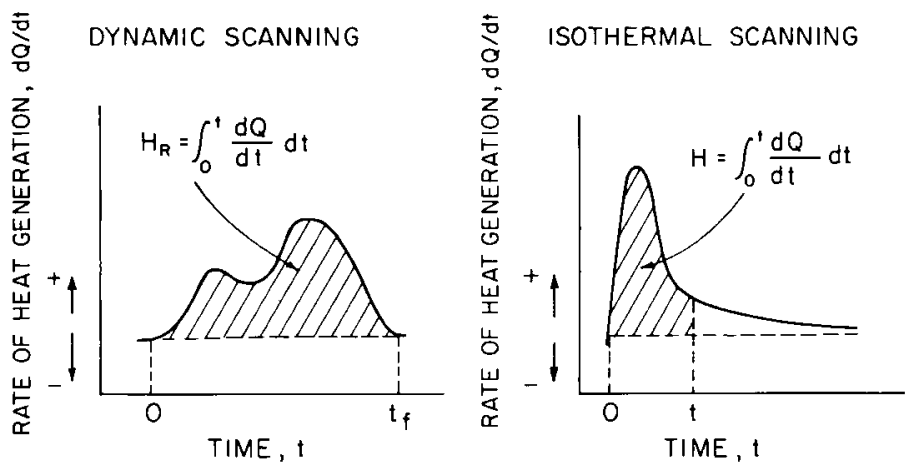

Figure 1. Illustration of rate of heat generation versus time curves measured by differential scanning calorimetry using dynamic scanning and isothermal scanning.

\section{Viscosity}

The viscosity was measured with a Rheometrics parallel disc and plate type mechanical spectrometer Model 605 [6]. The radius of the disc was $25 \mathrm{~mm}$ and the gap between the disc and the plate was $0.5 \mathrm{~mm}$. The disc oscillated at $1.6 \mathrm{~Hz}$. A data reduction and analyzing system built into the apparatus provided directly the complex viscosity [7] in poise as a function of time. The complex viscosity is assumed to be nearly the same as the shear viscosity $[7,8]$. The viscosity was measured at $375 \mathrm{~K}, 400 \mathrm{~K}$ and $425 \mathrm{~K}$. The time required in each test ranged from 2 hours (at $375 \mathrm{~K}$ ) to 30 minutes (at $425 \mathrm{~K}$ ). The time required to reach the steady state was about 3 minutes at all three temperatures.

\section{RESULTS}

The heat of reaction, the degree of cure, and the viscosity of Hercules 3501-6 resin were determined. A brief description of the resin is given in Table 1.

\section{Heat of Reaction}

The heat of reaction of the resin was determined from the results of the dynamic scanning measurements. The tests were repeated four times. A typical

Table 1. Composition of Hercules 3501-6 Resin. Amounts given are in parts per hundred resin [14]

\begin{tabular}{lr}
\hline Epoxide No. $1(\mathrm{H})$ & 100 \\
Curing Agent (DDS) & 44 \\
Epoxide No. 2 (H) & 16 \\
Epoxide No. $3(\mathrm{H})$ & 15 \\
BF $_{3}$ & 2 \\
\hline
\end{tabular}




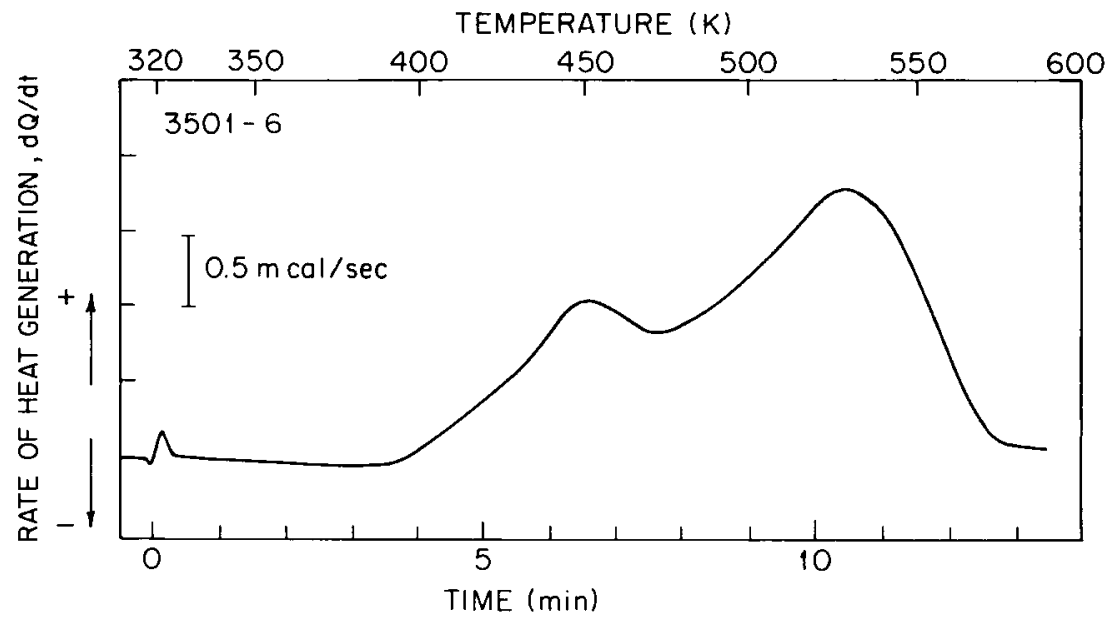

Figure 2. A typical rate of heat generation versus time curve provided by dynamic scanning experiments. Sample size $4.3 \mathrm{mg}$. Temperature scale constructed from the known value of the scanning rate which was $20 \mathrm{~K} / \mathrm{min}$.

result is shown in Figure 2. Note that there are two "humps" in the $d Q / d t$ versus time curve. These humps are caused by two different major reactions occurring during the cure. The measurements gave the following value for the heat of reaction

$$
H_{R}=473.6 \pm 5.4 \mathrm{~J} / \mathrm{g}
$$

\section{Degree of Cure}

The degree of cure $\alpha$ and the rate of degree of cure $d \alpha / d t$ were determined from the results of the isothermal scanning experiments. From the measured values of $d Q / d t$, the heat released $H$ up to time $t$, the degree of cure $\alpha$ at time $t$, and the rate of degree of cure $d \alpha / d t$ at time $t$ were calculated using Equations (2-5). The results are plotted in Figure 3.

Efforts were made to describe the $d \alpha / d t$ versus $\alpha$ data with a modified Arrhenius type equation. In this investigation, the following equations were found to describe the data well [9-11]

$$
\begin{aligned}
d \alpha / d t & =\left(K_{1}+K_{2} \alpha\right)(1-\alpha)(B-\alpha) & & \alpha \leq 0.3 \\
d \alpha / d t & =K_{3}(1-\alpha) & & \alpha>0.3
\end{aligned}
$$

where

$$
\begin{aligned}
& K_{1}=A_{1} \exp \left(-\Delta E_{1} / R T\right) \\
& K_{2}=A_{2} \exp \left(-\Delta E_{2} / R T\right) \\
& K_{3}=A_{3} \exp \left(-\Delta E_{3} / R T\right)
\end{aligned}
$$


Woo Il Lee, Alfred C. Loose, and George S. Springer

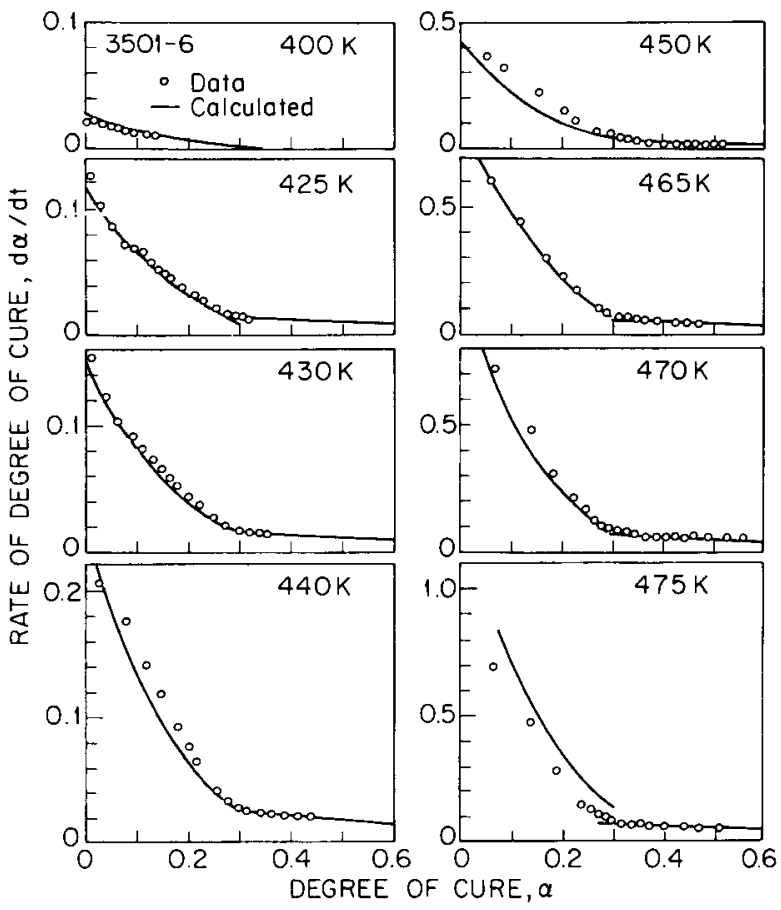

Figure 3. Rate of degree of cure versus degree of cure. Solid lines were calculated using Equation (6-8) and the constants given in Table 2. Note the difference in scales on the vertical axes of the figures on the left and on the right.

$A_{1}, A_{2}, A_{3}$ are the pre-exponential factors, $\Delta E_{1}, \Delta E_{2}, \Delta E_{3}$ are the activation energies, $R$ is the universal gas constant, and $T$ is the absolute temperature. $B$ is a constant independent of temperature. The constants $K_{1}, K_{2}$ and $B$ were determined by a non-linear least square curve fit to the $d \alpha / d t$ versus $\alpha$ data using the Levenberg-Marquardt algorithm [12]. This procedure gave the following value of $B$

$$
B=0.47 \pm 0.07
$$

Using the value $B=0.47$, the values of $K_{1}$ and $K_{2}$ were calculated by a linear least square curve fit to the data at $\alpha$ values less than 0.3 . The $K_{1}$ and $K_{2}$ values thus calculated are plotted in Figure 4. The value of $K_{3}$ was obtained by fitting a linear least square curve to the $d \alpha / d t$ versus $\alpha$ data at $\alpha$ values greater than 0.3 $(\alpha>0.3)$. The resulting $K_{3}$ values are also shown in Figure 4 .

The values of $A_{1}, A_{2}, A_{3}$ and $\Delta E_{1}, \Delta E_{2}, \Delta E_{3}$ were found by fitting straight lines to the $K_{1}, K_{2}, K_{3}$ versus $1 / T$ data. The values of these constants are listed in Table 2 . 
Heat of Reaction, Degree of Cure, and Viscosity of Hercules 3501-6 Resin

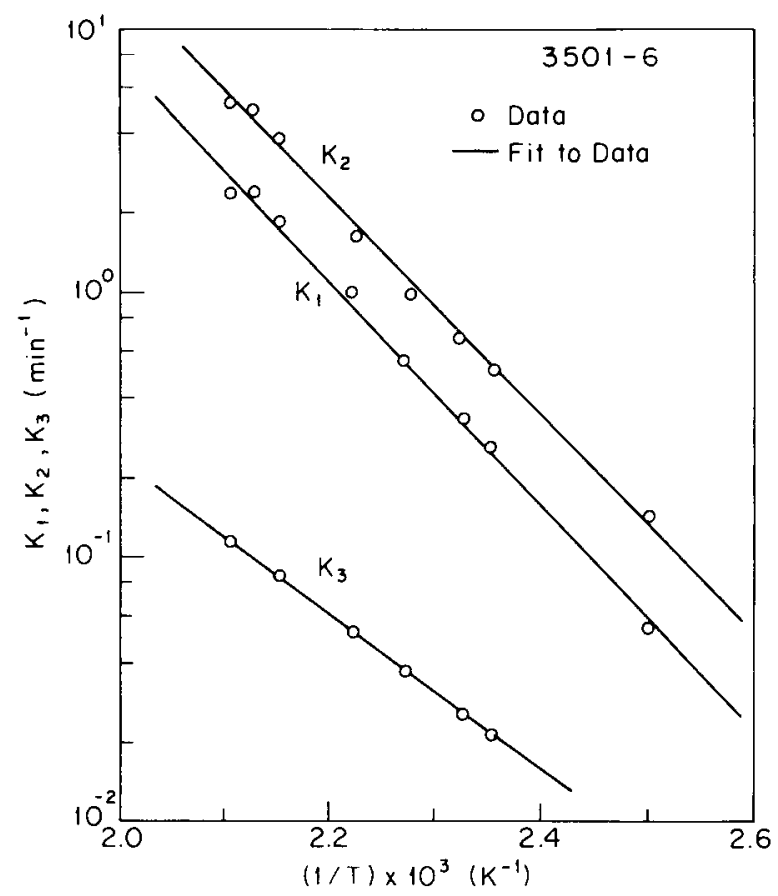

Figure 4. The parameters $K_{1}, K_{2}$ and $K_{3}$ appearing in Equations (6-8) as functions of inverse absolute temperature. Solid lines are least square fit to data.

The reaction rate is described by two different equations (Equations (6-7)). The results of the dynamic scanning measurement showed that there are at least two dominant reactions occurring during the cure. These reactions (represented by the two "humps" in Figure 1) cannot be correlated by a single equation, but must be described by different equations. This is the reason for the two equations employed here.

Comparisons between the measured values of $d \alpha / d t$ and the values calculated by Equations (6-8) are shown in Figure 3.

Table 2. The value of the constants in Equation (8)

$$
\begin{aligned}
A_{1} & =2.101 \times 10^{9} \mathrm{~min}^{-1} \\
A_{2} & =-2.014 \times 10^{9} \mathrm{~min}^{-1} \\
A_{3} & =1.960 \times 10^{5} \mathrm{~min}^{-1} \\
\Delta E_{1} & =8.07 \times 10^{4} \mathrm{~J} / \mathrm{mol} \\
\Delta E_{2} & =7.78 \times 10^{4} \mathrm{~J} / \mathrm{mol} \\
\Delta E_{3} & =5.66 \times 10^{4} \mathrm{~J} / \mathrm{mol}
\end{aligned}
$$


By integrating Equation (6) and Equation (7), the following relationships between $\alpha$ and $t$ are obtained

$$
\begin{aligned}
& t=\frac{a}{K_{2}} \ln \left(1+\frac{K_{2}}{K_{1}} \alpha\right)-b \ln (1-\alpha)-c \ln \left(1-\frac{\alpha}{B}\right) \\
& t=-\frac{1}{K_{3}} \ln \left[\frac{1-\alpha}{0.7}\right]+t_{c}
\end{aligned}
$$

where

$$
\begin{aligned}
& a=K_{2}^{2}(B-1) / d \\
& b=\left(K_{1}+K_{2} B\right) / d \\
& c=-\left(K_{1}+K_{2}\right) / d \\
& d=\left(K_{1}+K_{2}\right)\left\{K_{1} B^{2}+\left(K_{1}-K_{2}\right) B-K_{1}\right\}
\end{aligned}
$$

and $t_{c}$ is the time given by Equation (10) for $\alpha=0.3\left(t=t_{c}\right.$ at $\left.\alpha=0.3\right)$.

\section{Viscosity}

The measured values of viscosity $\mu$ as functions of time are given in Figure 5 . Since the degree of cure $\alpha$ is also a function of time (see Equations (10)and (11)), the viscosity can also be expressed in terms of $\alpha$. The viscosities as functions of $\alpha$

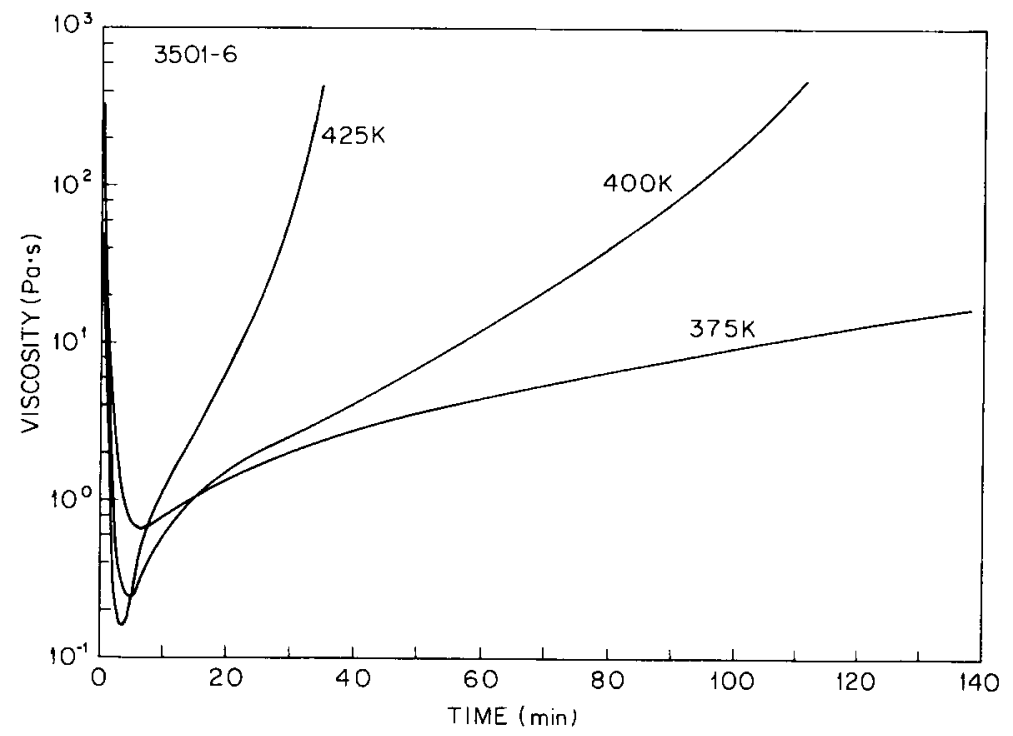

Figure 5. Measured viscosity as a function of time. 
are given in Figure 6. An expression was sought to correlate the data given in Figure 6. The following equation was found to represent the data [13]

$$
\mu=\mu_{\infty} \exp (U / R T+K \alpha)
$$

where $\mu_{\infty}$ is a constant, $U$ is the activation energy for viscosity, and $K$ is a constant which is independent of temperature.

The constants in Equation (13) were determined as follows. Equation (13) was written in the form

$$
\ln \mu=A+K \alpha
$$

where

$$
A=\ln \mu_{\infty}+U / R T
$$

The constant $K$ and the parameter $A$ were found by fitting a linear least square curve to the $\mu$ versus $\alpha$ data generated at a constant temperature. This procedure yielded the following value of $K$ :

$$
K=14.1 \pm 1.2
$$

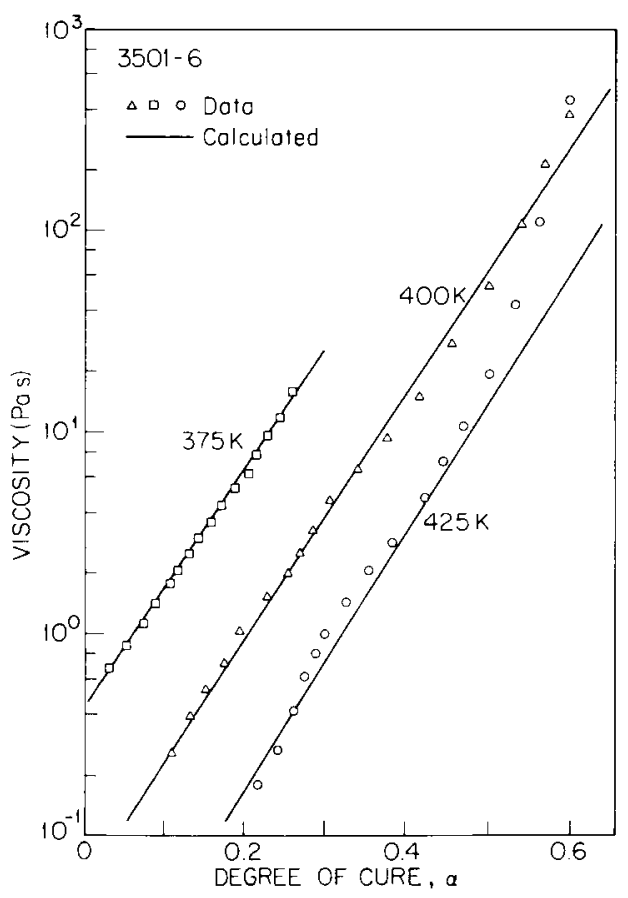

Figure 6. Viscosity as a function of degree of cure. Solid lines were calculated using Equation (13) and the constants given in Equations (16) and (17). 
The values of $A$ are shown as a function of the inverse of the absolute temperature in Figure 7. A straight line fit to the $A$ versus $1 / T$ data provided the following values of $\mu_{\infty}$ and $U$

$$
\begin{aligned}
\mu_{\infty} & =7.93 \times 10^{-14} \mathrm{~Pa} \cdot \mathrm{s} \\
U & =9.08 \times 10^{4} \mathrm{~J} / \mathrm{mol}
\end{aligned}
$$

The viscosities calculated using Equations (13) together with the above constants are also shown in Figure 6. As can be seen there is reasonable agreement between the data and the values given by Equation (13) when the degree of cure is less than about $0.5(\alpha<0.5)$. At degrees of cure higher than 0.5 the resin gels and Equation (13) becomes inapplicable. This is likely the reason for the discrepancy between the data and the calculated viscosity values at $\alpha$ values higher than 0.5 .

Viscosities for the Hercules 3501-6 resin system were reported by Carpenter [14]. Carpenter measured viscosities at different rate of heat generations and reported the measured values of visocisity as functions of time. In order to illustrate the usefulness of the expression developed in this investigation in situations where the temperature is not constant, viscosities were calculated for the heating rates employed by Carpenter. The calculations were performed as follows.

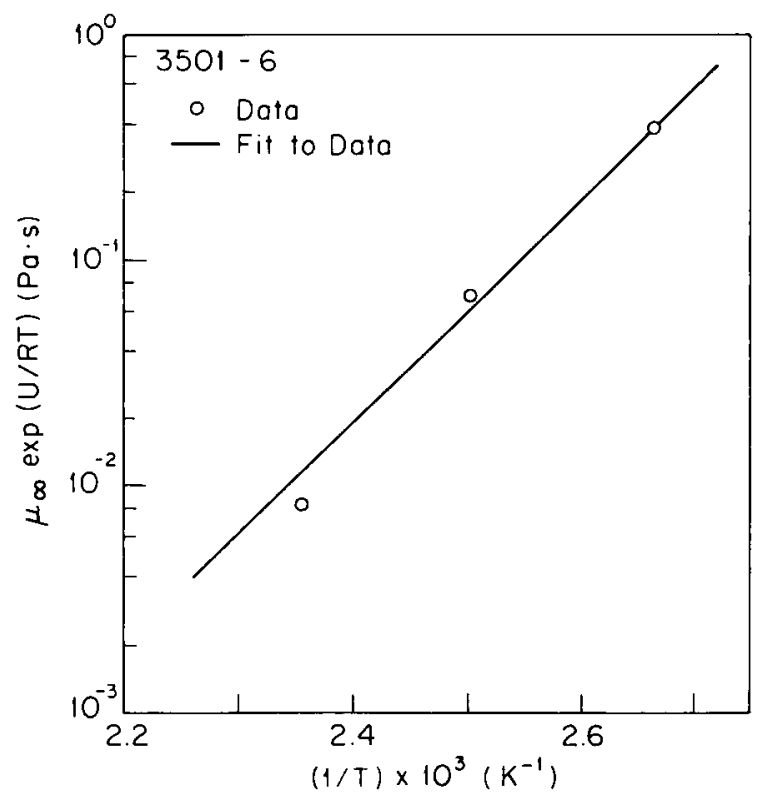

Figure 7. The parameter $\mu_{\infty} \exp (U / R T)$ appearing in Equation (13) as a function of inverse absolute temperature. Solid lines are least square fit to the data. 
1) At every 0.5 seconds, the temperature was calculated from the heating rate $d T / d t$ employed in Carpenter's measurement (Figure 8).

2) At each temperature, $d \alpha / d t$ was calculated using Equations (6) and (7), and the values of $\alpha$ were determined by numerical integration of these equations.

3) From the known values of the temperature $T$ and the degree of cure $\alpha$, the viscosities were calculated using Equation (13).

The viscosity values calculated according to this procedure are compared to Carpenter's data in Figure 8. As can be seen, the calculated viscosities and the data reported by Carpenter agree reasonably well, even though the calculations are based on viscosity measurements performed under isothermal conditions, whereas Carpenter's data were obtained under non-isothermal conditions.

\section{CONCLUDING REMARKS}

The values of the degree of cure $\alpha$, the rate of degree of cure $d \alpha / d t$, and the viscosity $\mu$ measured in this investigation could be used directiy in modelling the cure process of fiber reinforced composites employing Hercules 3501-6 resin.

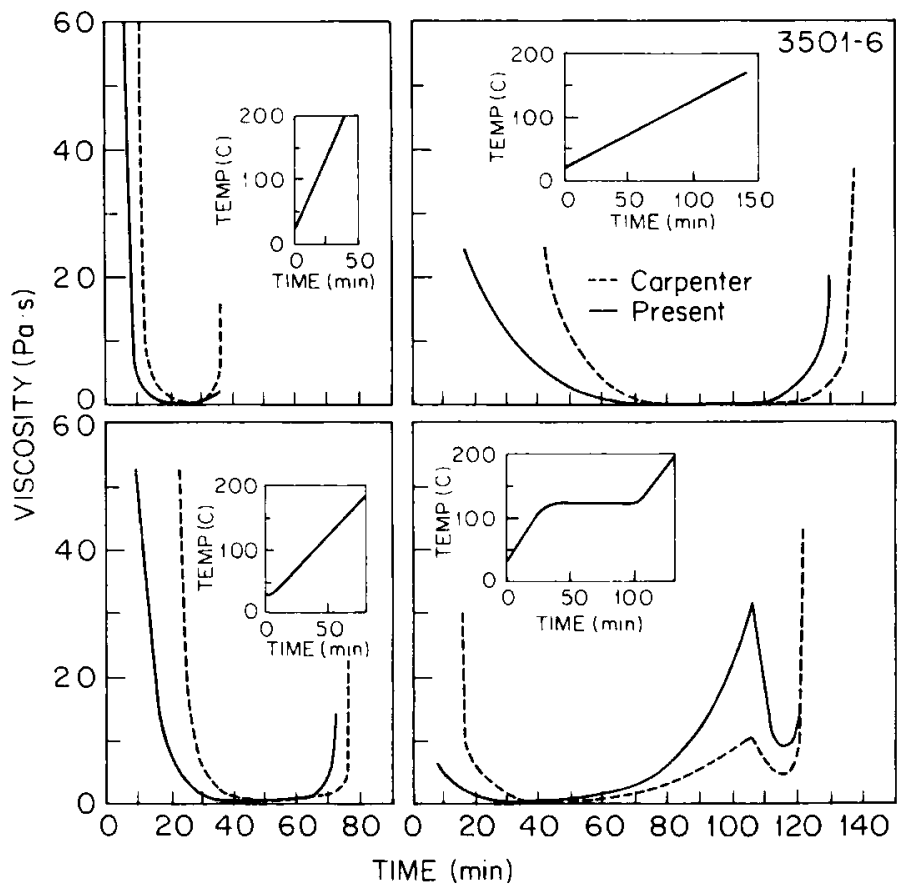

Figure 8. Comparison between the viscosities measured by Carpenter [14] and calculated by the present correlation (Equation (13)) for the temperature histories employed in Carpenter's measurements. 
Such models generally require computer solutions of the governing equations describing the flow and the heat transfer processes. In such computer calculations, it is often advantageous to use analytical expressions for $d \alpha / d t, \alpha, \mu$ instead of the data presented in tabular form. The expressions developed in this investigation should be adequate for that purpose.

\section{ACKNOWLEDGEMENTS}

This work was supported by the U.S. Air Force Systems Command, Materials Laboratory, Wright-Patterson Air Force Base, Dayton, Ohio with Dr. S. W. Tsai acting as project engineer.

\section{REFERENCES}

1. Aung, W., "Calculation of Resin Temperature and Resin Flow Rate during Multilayer Board Lamination," International Electronic Packaging and Production Conf. Proc. of the Tech. Programme INTERNEPCON EUROPE 1973, Brussels (1973).

2. Loos, A. C. and Springer, G. S., "Calculation of Cure Process Variables during Cure of Graphite-Epoxy Composites," in Composite Materials, Quality Assurance and Processing (C.E. Browning, ed.). ASTM STP 797, 1983, pp. 110-118.

3. Springer, G. S., "A Model of the Curing Process of Epoxy Matrix Composites," in Progress in Science and Engineering of Composites (T. Hayashi, K. Kowaka and S. Umekawa, eds.), Japan Society for Composite Materials, 1982, pp. 23-35.

4. Model DSC-2 Differential Scanning Calorimeter Instructions Manual, Perkin-Elmer Corporation (1972).

5. Fava, R. A., "Differential Scanning Calorimetry of Epoxy Resins," Polymer, Vol. 13, 1968, pp. 137-151.

6. Macosko, C. and Starita, J. M., "Polymer Characteristics with a New Rheometer," $S P E$ Tech. Papers, Vol. 17, 1971, pp. 595-600.

7. Rosen, S. L., Fundamental Principles of Polymeric Materials, Wiley, 1982, Chap. 18.

8. Cox, W. P. and Merz, E. H., "Correlation of Dynamic and Steady Flow Viscosities," J. Polym. Sci., Vol. 28, 1958, pp. 619-622.

9. Sourour, S. and Kamal, M. R., "Differential Scanning Calorimetry of Epoxy Cure: Isothermal Cure Kinetics," Thermochimica Acta, Vol. 14, 1976, pp. 41-59.

10. Prime, R. Bruce, "Differential Scanning Calorimetry of the Epoxy Cure Reaction," Polym. Eng. Sci., Vol. 13, 1973, pp. 365-371.

11. Pappalardo, L. T., "DSC Evaluation of Epoxy and Polyamide-Impregnated Laminates (Prepregs)," J. Appl. Polym. Sci., Vol. 21, 1977, pp. 809-820.

12. IMSL Library Reference Manual, International Mathematical and Statistical Libraries Incorporated, Houston, Texas, Vol. 3, 1980.

13. Stolin, A. M., Merzhanov, A. G. and Malkin, A. Ya, "Non-Isothermal Phenomena in Polymer Engineering and Science: A Review. Part II: Non-Isothermal Phenomena in Polymer Deformation," Polym. Eng. Sci., Vol. 19, 1979, pp. 1074-1080.

14. Carpenter, J. F., "Test Program Evaluation of Hercules 3501-6 Resin," Report No. N000 9-77-C-0155 McDonnell Aircraft Company, St. Louis, Missouri (1978). Also available from NTIS under order No. AD-A-054283, 1978. 